\title{
Harmonic vectors and matrix tree theorems
}

\author{
SidDHARTHA SAHI
}

This paper describes an explicit combinatorial formula for a harmonic vector for the Laplacian of a directed graph with arbitrary edge weights. This result was motivated by questions from mathematical economics, and the formula plays a crucial role in recent work of the author on the emergence of prices and money in an exchange economy.

It turns out that the formula is closely related to well-studied problems in graph theory, in particular to the so-called weighted matrix tree theorem due to $\mathrm{W}$. Tutte and independently to R. Bott and J. Mayberry. As a further application of our considerations, we obtain a short new proof of both the matrix tree theorem as well as its generalization due to S. Chaiken.

MSC 2010 SubJeCt CLASSifications: Primary 05A15; secondary 05C22, 05C30, 91B24, 91B26.

KEYWORDS AND PHRASES: Market mechanisms, discrete Laplacian, matrix tree theorem, all minors.

\section{Introduction}

In this paper we prove a new result in graph theory that was motivated by considerations in mathematical economics, more precisely, by the problem of price formation in an exchange economy [4]. The aggregate demand/supply in the economy is described by an $n \times n$ matrix $A=\left(a_{i j}\right)$ where $a_{i j}$ is the amount of commodity $j$ that is on offer for commodity $i$. In this context one defines a market-clearing price vector to be a vector $p$ with strictly positive components $p_{i}$, which satisfies the equation

$$
\sum_{j} a_{i j} p_{j}=\sum_{j} a_{j i} p_{i} \text { for all } i .
$$

The left side of (1) represents the total value of all commodities being offered for commodity $i$, while the right side represents the total value of commodity $i$ in the market. It was shown in [4] that if the matrix $A$ is irreducible, i.e. if it cannot be permuted to block upper-triangular form, then (1) admits a positive solution vector $p$, which is unique up to a positive multiple. 
The primary purpose of the present paper is to describe an explicit combinatorial formula for $p$. The formula and its proof are completely elementary, but nonetheless the result seems to be new. This formula plays a crucial role in [5], which seeks to address a fundamental question in mathematical economics: How do prices and money emerge in a barter economy? We show in [5] that among a reasonable class of exchange mechanisms, trade via a commodity money, even in the absence of transactions costs, minimizes complexity in a very precise sense.

It turns out however that equation (1) is closely related to well-studied problems in graph theory, in particular to the so-called matrix tree theorems. Therefore as an additional application of our formula, we give an elementary proof of the matrix tree theorem of $\mathrm{W}$. Tutte [6], which was independently discovered by R. Bott and J. Mayberry [2] coincidentally also in an economic context. With a little additional effort, we also obtain a short new proof of S. Chaiken's generalization of the matrix tree theorem [3].

\section{Harmonic vectors}

We first give a slight reformulation and reinterpretation of equation (1) in standard graph-theoretic language. Let $G$ be a simple directed graph (digraph) on the vertices $1,2, \ldots, n$, with weight $a_{i j}$ attached to the edge $i j$ from $i$ to $j$. The weighted adjacency matrix of $G$ is the $n \times n$ matrix $A=\left(a_{i j}\right)$, where $a_{i j}=0$ for missing edges. The weighted degree matrix $D$ is the diagonal matrix with diagonal entries $\left(d_{1}, \ldots, d_{n}\right)$, where $d_{i}=\sum_{j} a_{j i}$ is the weighted in-degree of the vertex $i$. The Laplacian of $G$ is the matrix $L=D-A$ and we say that a vector $\mathbf{x}=\left(x_{i}\right)$ is harmonic if $\mathbf{x}$ is a null vector of $L$, i.e. if it satisfies

$$
L \mathbf{x}=\mathbf{0}
$$

It is easy to see that equation (1) is equivalent to equation (2), i.e. the market-clearing condition is the same as harmonicity of $p$.

To describe our construction of a harmonic vector, we introduce some terminology. A directed tree, also known as an arborescence, is a digraph with at most one incoming edge $i j$ at each vertex $j$, and whose underlying undirected graph is acyclic and connected (i.e. a tree). Following the edges backwards from any vertex we eventually arrive at the same vertex called the root. Dropping the connectivity requirement leads to the notion of a directed forest, which is simply a vertex-disjoint union of directed trees. We define a dangle to be a digraph $D$ that is an edge-disjoint union of a directed forest 
$F$ and a directed cycle $C$ linking the roots of $F$; note that $D$ determines $C$ and $F$ uniquely, the former as its unique simple cycle.

In the context of the digraph $G$, we will use the term $i$-tree to mean a directed spanning tree of $G$ with root $i$, and $i$-dangle to mean a spanning dangle whose cycle contains $i$. We define the weight $w t(\Gamma)$ of a subgraph $\Gamma$ of $G$ to be the product of weights of all the edges of $\Gamma$, and we define the weight vector of $G$ to be $\mathbf{w}=\left(w_{i}\right)$ where $w_{i}$ is the weighted sum of all $i$-trees.

Theorem 1. The weight vector of a digraph is harmonic.

Proof. If $\Gamma$ is an $i$-dangle in $G$ with cycle $C$, and $i j$ and $k i$ are the unique outgoing and incoming edges at $i$ in $C$, then deleting one of these edges from $\Gamma$ gives rise to an $j$-tree and a $i$-tree, respectively. The dangle can be recovered uniquely from each of the two trees by reconnecting the respective edges; thus, writing $\mathcal{T}_{i}$ for the set of $i$-trees, we obtain bijections from the set of $i$-dangles to each of the following sets

$$
\left\{(i j, t): t \in \mathcal{T}_{j}\right\}, \quad\left\{(k i, t): t \in \mathcal{T}_{i}\right\}
$$

where $i j$ and $k i$ range over all outgoing and incoming edges at $i$ in $G$.

Thus if $v_{i}$ is the weighted sum of all $i$-dangles, we get

$$
\sum_{j} a_{i j} w_{j}=v_{i}=\sum_{k} a_{k i} w_{i} .
$$

Rewriting this we get $A \mathbf{w}=D \mathbf{w}$, and hence $(D-A) \mathbf{w}=\mathbf{0}$, as desired.

\section{The matrix tree theorem}

In this section we use Theorem 1 to derive the weighted matrix tree theorem due to [6] (see also [2]). This is the following formula for the cofactors of the Laplacian $L$, which generalizes a classical formula of Kirchoff for the number of spanning trees in an undirected graph.

Theorem 2. The ij-th cofactor of the Laplacian $L$ is given by

$$
c_{i j}(L)=\sum_{t \in \mathcal{T}_{j}} w t(t) \text { for all } i, j .
$$

We will prove this in a moment after some discussion on cofactors. 


\subsection{Interlude on cofactors}

We recall that $i j$-th cofactor of an $n \times n$ matrix $X$ is

$$
c_{i j}(X)=(-1)^{i+j} \operatorname{det} X_{i j}
$$

where $X_{i j}$ is the matrix obtained from $X$ by deleting row $i$ and column $j$. The adjoint of $X$ is the $n \times n$ matrix adj $(X)$ whose $i j$-th entry is $c_{j i}(X)$.

Lemma 3. If $\operatorname{det} X=0$ then the columns of adj $(X)$ are null vectors of $X$; moreover these are the same null vector if the columns of $X$ sum to 0.

Proof. By standard linear algebra we have $X \operatorname{adj}(X)=(\operatorname{det} X) I_{n}$. If $\operatorname{det} X=0$ then $X \operatorname{adj}(X)$ is the zero matrix, which implies the first part. For the second part we note that if $X$ has zero column sums then necessarily $\operatorname{det} X=0$. In view of the first part it suffices to show that $c_{i j}(L)=c_{i+1, j}(L)$ for all $i, j$; or equivalently that

$$
\operatorname{det} L_{i j}+\operatorname{det} L_{i+1, j}=0 \text {. }
$$

The left side above equals det $P$, where $P$ is obtained from $L$ by deleting column $j$ and replacing rows $i$ and $i+1$ by the single row consisting of their sum. But $P$ too has zero column sums, and so $\operatorname{det} P=0$.

\subsection{Proof of the matrix tree theorem}

Proof. It suffices to prove Theorem 2 for the complete simple digraph $G_{n}$ on $n$ vertices, with edge weights $\left\{a_{i j} \mid i \neq j\right\}$ regarded as variables, and we work over the field of rational functions $\mathbb{C}\left(a_{i j}\right)$. The Laplacian $L$ has zero column sums by construction, and so by the previous lemma, $c_{j}:=c_{i j}(L)$ is independent of $i$ and the vector $\mathbf{c}=\left(c_{1}, \ldots, c_{n}\right)^{t}$ is a null vector for $L$. To complete the proof it suffices to show that the null vectors $\mathbf{c}$ and $\mathbf{w}$ are equal. Now the null space of $L$ is 1 -dimensional since $c_{i j}(L) \neq 0$, and hence

$$
c_{i} w_{j}=c_{j} w_{i} \text { for all } i, j \text {. }
$$

Note that $c_{j}$ and $w_{j}$ belong to the polynomial ring $\mathbb{C}\left[a_{i j}\right]$. We claim that the polynomials $c_{j}$ are distinct and irreducible. Consider first $c_{n}=\operatorname{det} B$ where $B=L_{n n}$ has entries

$$
b_{i j}=\left\{\begin{array}{cl}
-a_{i j} & \text { if } i \neq j \\
a_{n j}+\sum_{k=1}^{n-1} a_{k j} & \text { if } i=j
\end{array} \quad ; \quad \text { for } 1 \leq i, j \leq n-1\right.
$$


This is an invertible $\mathbb{C}$-linear map relating $\left\{b_{i j}\right\}$ to the $(n-1)^{2}$ variables

$$
\left\{a_{i j} \mid 1 \leq i \leq n, 1 \leq j \leq n-1, i \neq j\right\}
$$

which occur in $c_{n}$. Thus the irreducibility of $c_{n}$ follows from the irreducibility of the determinant as a polynomial in the matrix entries [1]. The argument for the other $c_{i}$ is similar, and their distinctness is obvious.

Since $c_{i}$ and $c_{j}$ are distinct and irreducible, we conclude from formula (3) that $c_{i}$ divides $w_{i}$. Since $c_{i}$ and $w_{i}$ both have total degree $n-1$, we conclude that $w_{i}=\alpha c_{i}$ for some $\alpha \in \mathbb{C}$. To prove that $\alpha=1$, it suffices to note that the monomial $m_{i}=\prod_{j \neq i} a_{i j}$ occurs in both $c_{i}$ and $w_{i}$ with coefficient 1 .

\section{The all minors theorem}

The all minors theorem [3] is a formula for $\operatorname{det} L_{I J}$, where $L_{I J}$ is the submatrix of $L$ obtained by deleting rows $I$ and columns $J$. It turns out this follows from Theorem 2 by a specialization of variables. We will state and prove this below after a brief discussion on signs of permutations and bijections.

\subsection{Interlude on signs}

Let $I, J$ be equal-sized subsets of $\{1, \ldots, n\}$ and let $\Sigma_{I}, \Sigma_{J}$ denote the sums of their elements. If $\beta: J \rightarrow I$ is a bijection, we write $i n v(\beta)$ for the number of inversions in $\beta$, i.e. pairs $j<j^{\prime}$ in $J$ such that $\beta(j)>\beta\left(j^{\prime}\right)$ and we define

$$
\varepsilon(\beta)=(-1)^{i n v(\beta)+\Sigma_{I}+\Sigma_{J}} .
$$

Note that if $J=I$ then $\varepsilon(\sigma)=(-1)^{i n v(\sigma)}$ is the sign of $\sigma$ as a permutation.

Lemma 4. If $\beta: J \rightarrow I, \alpha: I \rightarrow H$ are bijections then $\varepsilon(\alpha \beta)=\varepsilon(\alpha) \varepsilon(\beta)$.

Proof. This follows by combining the following mod 2 congruences

$$
\begin{aligned}
\Sigma_{H}+\Sigma_{I}+\Sigma_{I}+\Sigma_{J} & \equiv \Sigma_{H}+\Sigma_{J} \\
i n v(\alpha)+i n v(\beta) & \equiv i n v(\alpha \beta)
\end{aligned}
$$

the first of which is obvious. To establish the second congruence we replace $\alpha, \beta$ by the permutations $\lambda \alpha, \beta \mu$ of $I$, where $\lambda: H \rightarrow I, \mu: I \rightarrow J$ are the unique order-preserving bijections; this does not affect inv $(\alpha)$ etc., and reduces the second congruence to a standard fact about permutations. 
The meaning of $\varepsilon(\beta)$ is clarified by the following result. For a bijection $\beta: J \rightarrow I$ and any $n \times n$ matrix $X$, let $X_{\beta}$ be the matrix obtained from $X$ by replacing, for each $j \in J$, the $j$ th column of $X$ by the unit vector $\mathbf{e}_{\beta(j)}$.

Lemma 5. We have $\operatorname{det} X_{\beta}=\varepsilon(\beta) \operatorname{det} X_{I J}$.

Proof. If $\sigma$ is a permutation of $I$ then by the previous lemma, and standard properties of the determinant, we have

$$
\varepsilon(\sigma \beta)=\varepsilon(\sigma) \varepsilon(\beta), \operatorname{det} X_{\sigma \beta}=\varepsilon(\sigma) \operatorname{det} X_{\beta} .
$$

Thus replacing $\beta$ by a suitable $\sigma \beta$, we may assume $i n v(\beta)=0$ and write

$$
I=\left\{i_{1}<\cdots<i_{p}\right\}, J=\left\{j_{1}<\cdots<j_{p}\right\} \text { with } \beta\left(j_{k}\right)=i_{k} \text { for all } k .
$$

The lemma now follows from the identity

$$
\operatorname{det} X_{\beta}=(-1)^{i_{p}+j_{p}} \cdots(-1)^{i_{1}+j_{1}} \operatorname{det} X_{I J}=(-1)^{\Sigma_{I}+\Sigma_{J}} \operatorname{det} X_{I J}
$$

obtained by iteratively expanding $\operatorname{det} X_{\beta}$ along columns $j_{p}, \ldots, j_{1}$.

\subsection{Directed forests}

Let $\mathcal{F}(J)$ be the set of all directed spanning forests $f$ of $G$ with root set $J$. Let $\mathcal{F} \subset \mathcal{F}(J)$ be the subset consisting of those forests $f$ such that each tree of $f$ contains a unique vertex of $I$. Note that the trees of $f \in \mathcal{F}$ give a bijection $\beta_{f}: J \rightarrow I$. The all minors theorem is the following formula [3].

Theorem 6. We have $\operatorname{det} L_{I J}=\sum_{f \in \mathcal{F}} \varepsilon\left(\beta_{f}\right) \mathrm{wt}(f)$.

We fix a bijection $\beta: J \rightarrow I$ and define $\sigma_{f}=\beta^{-1} \beta_{f}: J \rightarrow J$. In view of Lemmas 4 and 5 , it suffices to prove the following reformulation of the previous theorem.

Theorem 7. We have $\operatorname{det} L_{\beta}=\sum_{f \in \mathcal{F}} \varepsilon\left(\sigma_{f}\right) \mathrm{wt}(f)$.

Proof. As usual it is enough to treat the complete digraph $G_{n}$ with arbitrary edge weights $a_{i j}$. We fix an index $j_{0} \in J$ and put $i_{0}=\beta\left(j_{0}\right), J_{0}=J \backslash\left\{j_{0}\right\}$. We now consider a particular specialization $\bar{a}_{i j}$ of $a_{i j}$, and the entries $\bar{l}_{i j}$ of the specialized Laplacian $\bar{L}$. For $j \notin J_{0}$ we set $\bar{a}_{i j}=a_{i j}$ and hence $\bar{l}_{i j}=a_{i j}$; while for $j \in J_{0}$ we set

$$
\bar{a}_{i j}=\left\{\begin{array}{cl}
1 & \text { if } i=i_{0} \\
-1 & \text { if } i=\beta(j) \\
0 & \text { otherwise }
\end{array} \Longrightarrow \bar{l}_{i j}=\left\{\begin{array}{cl}
-1 & \text { if } i=i_{0} \\
1 & \text { if } i=\beta(j) \\
0 & \text { otherwise }
\end{array}\right.\right.
$$


Note that $\bar{L}$ and $L_{\beta}$ have the same entries outside of row $i_{0}$ and column $j_{0}$; hence we get $\operatorname{det} L_{\beta}=c_{i_{0} j_{0}}\left(L_{\beta}\right)=c_{i_{0} j_{0}}(\bar{L})$ and it remains to show that

$$
c_{i_{0} j_{0}}(\bar{L}) \stackrel{?}{=} \sum_{f \in \mathcal{F}} \varepsilon\left(\sigma_{f}\right) \mathrm{wt}(f) .
$$

Specializing Theorem 2 we get

$$
c_{i_{0} j_{0}}(\bar{L})=\sum_{f \in \mathcal{F}(J)} \psi(f) \mathrm{wt}(f), \psi(f):=\sum_{t \in \mathcal{A}_{f}}(-1)^{p(t)},
$$

where $\mathcal{A}_{f}$ is the set of $j_{0}$-trees $t$ such that for each $j \in J_{0}$ the unique edge $i j$ in $t$ satisfies $i=i_{0}$ or $i=\beta(j)$, and for which deleting all such edges from $t$ yields the forest $f$; and where $p(t)$ is the number of edges in $t$ of type $i_{0} j$, $j \in J_{0}$. Therefore to prove equality in formula (5) it suffices to show

$$
\psi(f) \stackrel{?}{=}\left\{\begin{array}{cc}
0 & \text { if } f \notin \mathcal{F} \\
\varepsilon\left(\sigma_{f}\right) & \text { if } f \in \mathcal{F}
\end{array} .\right.
$$

First suppose $f \notin \mathcal{F}$. In this case if $t \in \mathcal{A}_{f}$ there is some $j \in J_{0}$ such that the $j$-subtree contains no $I$ vertex. Choose the largest such $j$ and change the edge $i j$, from $i=i_{0}$ to $i=\beta(j)$ or vice versa. This is a sign-reversing involution on $\mathcal{A}_{f}$ and hence we get $\psi(f)=0$.

Now let $f \in \mathcal{F}$, and for each subset $S \subset J_{0}$ consider the graph obtained from $f$ by adding the edges $i_{0} j$ for $j \in S$, and $\beta(j) j$ for $j \in J_{0} \backslash S$. This graph is a tree in $\mathcal{A}_{f}$ iff $S$ meets every cycle $c$ of the permutation $\sigma_{f}$ of $J$, and is disconnected otherwise. Thus a tree $t \in \mathcal{A}_{f}$ is prescribed uniquely by choosing, for each cycle $c$ of $\sigma_{f}$, a nonempty subset $S_{c}$ of its vertex set $J_{c}$. By definition we have $(-1)^{p(t)}=\prod_{c}(-1)^{\left|J_{c}\right|-\left|S_{c}\right|}$, and so $\psi(f)$ factors as

$$
\psi(f)=\prod_{c} \psi(c), \psi(c):=\sum_{J_{c} \supseteq S_{c} \neq \emptyset}(-1)^{\left|J_{c}\right|-\left|S_{c}\right|} .
$$

Now we get $\psi(c)=(-1)^{\left|J_{c}\right|-1}$ using the elementary identity

$$
\sum_{k=1}^{m}\left(\begin{array}{c}
m \\
k
\end{array}\right)(-1)^{m-k}=(1-1)^{m}-(-1)^{m}=(-1)^{m-1} .
$$

Thus $\psi(f)$ agrees with the standard formula $\prod_{c}(-1)^{\left|J_{c}\right|-1}$ for $\varepsilon\left(\sigma_{f}\right)$. 


\section{References}

[1] M. Bocher; Introduction to Higher Algebra. Dover Publications Inc., New York, 1964, pp. 176. MR0172882

[2] R. Bott and J. Mayberry; Matrices and trees, pp. 391-400 in Economic activity analysis. Edited by O. Morgenstern. John Wiley and Sons (New York), 1954. MR0067067

[3] S. Chaiken; A combinatorial proof of the all minors matrix tree theorem, SIAM J. Alg. Disc. Methd. 3 (1982), 319-329. MR0666857

[4] P. Dubey and S. Sahi; Price-mediated trade with quantity signals: an axiomatic approach, J. Math. Econ. 39 (2003), 377-390. MR1996482

[5] P. Dubey, S. Sahi, and M. Shubik; Minimally Complex Exchange Mechanisms: Emergence of Prices, Markets, and Money, preprint, available online as Cowles Foundation Discussion Paper 1945, http://cowles.econ.yale.edu/P/cd/d19a/d1945.pdf.

[6] W. Tutte; The dissection of equilateral triangles into equilateral triangles, Proc. Cambridge Philos. Soc. 44 (1948), 463-482. MR0027521

Siddhartha SAHI

Mathematics Department

Rutgers UNIVERSITY

New Brunswick, NJ

USA

E-mail address: sahi@math.rutgers.edu

Received September 16, 2013 